\title{
HSP70 and modified HPV 16 E7 fusion gene without the addition of a signal peptide gene sequence as a candidate therapeutic tumor vaccine
}

\author{
JINBAO ZONG ${ }^{1,2}$, CHANGYUAN WANG ${ }^{1,3}$, QINGYONG WANG $^{1}$, \\ QINGLIN PENG ${ }^{1}$, YUFEI XU ${ }^{1}$, XIXIU XIE ${ }^{1}$ and XUEMEI XU ${ }^{1}$ \\ ${ }^{1}$ Department of Biophysics and Structural Biology, Institute of Basic Medical Sciences, Chinese Academy \\ of Medical Sciences, School of Basic Medicine Peking, Union Medical College, Beijing 100005; ${ }^{2}$ Clinical Laboratory, \\ The Affiliated Hospital of Medical College, Qingdao University, Qingdao 266003, P.R. China
}

Received August 2, 2013; Accepted September 6, 2013

DOI: $10.3892 / o r .2013 .2742$

\begin{abstract}
Millions of women are currently infected with high-risk human papillomavirus (HPV), which is considered to be a major risk factor for cervical cancer. Thus, it is urgent to develop therapeutic vaccines to eliminate the established infections or HPV-related diseases. In the present study, using the Mycobacterium tuberculosis heat shock protein 70 (MtHSP70) gene linked to the modified HPV 16 E7 (mE7) gene, we generated two potential therapeutic HPV DNA vaccines, mE7/MtHSP70 and SigmE7/MtHSP70, the latter was linked to the signal peptide gene sequence of human CD33 at the upstream of the fusion gene. We found that vaccination with the mE7/MtHSP70 DNA vaccine induced a stronger E7-specific $\mathrm{CD}^{+} \mathrm{T}$ cell response and resulted in a more significant therapeutic effect against E7-expressing tumor cells in mice. Our results demonstrated that HSP70 can play a more important role in mE7 and MtHSP70 fusion
\end{abstract}

Correspondence to: Dr Xuemei Xu, Department of Biophysics and Structural Biology, Institute of Basic Medical Sciences, Chinese Academy of Medical Sciences, School of Basic Medicine, Peking Union Medical College, Beijing 100005, P.R. China

E-mail: xuemeixu@vip.sina.com

Present address: ${ }^{3}$ Department of Dermatology, Qingdao Municipal Hospital, Qingdao 266001, P.R. China

Abbreviations: HPV, human papillomavirus; CTL, cytotoxic T lymphocyte; MHC, major histocompatibility complex; HSP, heat shock protein; APC, antigen-presenting cell; DC, dendritic cell; mE7, modified and optimized HPV16 E7 gene; mE7/MtHSP70, modified E7 linked with Mycobacterium tuberculosis HSP70; SigmE7/MtHSP70, modified E7 linked with Mycobacterium tuberculosis HSP70 attached with CD33 signal peptide; FCS, fetal calf serum; OPD, $o$-phenylenediamine; TLR, toll-like receptor

Key words: heat shock protein, human papillomavirus, E7, signal peptide, tumor vaccine
DNA vaccine without the help of a signal peptide. This may facilitate the use of HSP70 and serve as a significant reference for future study.

\section{Introduction}

Cervical cancer is the third most common cancer worldwide, and more than $85 \%$ of cervical cancer cases occur in developing countries (1). It is well known that infection of the oncogenic type of HPVs, particularly HPV 16, is an etiologic factor of cervical cancers (2,3). Although the two HPV major capsid protein L1 virus-like particle-based preventive vaccines have a remarkable safety profile and clinical efficacy against the HPV genotypes from which they were derived, they are not effective in the elimination of pre-existing infection and HPV-associated diseases $(4,5)$. Thus, it is urgent to develop therapeutic HPV vaccines. Since the HPV oncoprotein E7 is constitutively expressed in HPV-infected cells and cervical cancers, it has become an attractive target for the development of HPV therapeutic vaccines (6-9).

DNA vaccines have become an attractive approach for generating antigen-specific immunotherapy. Naked plasmid DNA can generate effective cytotoxic T lymphocytes (CTLs) and antibody responses by delivering foreign antigens to antigen-presenting cells (APCs) that stimulate $\mathrm{CD}^{+}$and $\mathrm{CD} 8^{+}$ $\mathrm{T}$ cells. They are easily prepared with high purity and stability and can be repeatedly administered (10). Several versatile immune stimulatory molecules have been used to overcome the weak immunogenicity of DNA vaccines, one of which is HSP70, a promising molecule due to its attractive adjuvant activity in enhancing antigen-specific immunity (11-13). Immunological functions of HSP70 can be categorized into chaperoning properties, cross-priming abilities and linking danger and pathogen recognition activities (14-20). Many HSP70-based therapeutic cancer vaccines have been reported $(11,13,21,22)$. We previously used human HSP70 or Mycobacterium tuberculosis HSP70 as a dendritic cell (DC)-targeting molecule to construct two HPV 16 mE7-based fusion DNA vaccines with a leader peptide gene sequence of CD33 (Sig) at the upstream of the fusion gene, SigmE7/MtHSP70 and 
SigmE7/HuHSP70 DNA. We found that vaccination of both the fusion DNA vaccines effectively enhanced the antitumor responses (23). The reason for choosing the leader sequence of the human CD33 as the signal peptide was that it has been used in tumor cell vaccines transfected with secretable HSP70 and displays enhanced antitumor efficacy (21). Currently, some HSP70-based HPV 16 E7 fusion DNA vaccines with or without an additional leading peptide sequence have shown promise in further clinical trials $(11,13,24)$. To investigate which strategy may facilitate HSP70 to function more efficiently in therapeutic fusion DNA vaccines, we considered two options: a strategy which constructs an antigen-HSP70 fusion gene alone or as an antigen-HSP70 fusion gene with a leading peptide.

In the present study, we constructed $\mathrm{mE7/MtHSP70} \mathrm{fused}$ tumor DNA vaccine and compared its potency in mice with pre-constructed SigmE7/MtHSP70 fusion DNA vaccine with a leader peptide gene sequence of the CD33 molecule at the upstream of the fusion gene. Our results showed that without the help of the CD33 signal peptide, the mE7/MtHSP70 fusion protein was efficiently release from cells, and vaccination of $\mathrm{mE7/MtHSP70} \mathrm{fusion} \mathrm{DNA} \mathrm{vaccine} \mathrm{induced} \mathrm{more} \mathrm{effec-}$ tive $\mathrm{CD}^{+} \mathrm{T}$ cell responses and antitumor effects than did SigmE7/MtHSP70 DNA. Our data suggest that without the help of a signal peptide, HSP70 can play a more powerful adjuvant role in antigen and HSP70 fused tumor DNA vaccine.

\section{Materials and methods}

Plasmid DNA constructs and preparation. We previously reported the modified HPV $16 \mathrm{E} 7$ gene with abolishment of its potential transformation activity and enhanced immunogenicity by a combination of gene shuffling, site-directed mutagenesis and codon optimization methods $(23,25) . \mathrm{mE} 7$ gene and SigmE7 containing SigCD33 were amplified by overlap PCR with the primers: 5'-CGAGTCGTGCGGCCGCC ACCATGCCGCTGCTGCTACTGCTGCCCCTGCTGTGG GCAG-3' (NotI), 5'-CTGCCCCTGCTGTGGGCAGGGGC CCTGGCTATGATGGATCTGCTCATGGGCAC-3' and 5'-GCTCTAGAGCGGTAGTCTCGGGCTGCAG-3' (XbaI). We digested Sig mE7 with NotI and $X b a I$ and ligated it to NotI/XbaI-digested pVR1012 to generate pVR1012-SigmE7. To generate pVR1012-SigmE7/MtHSP70, MtHSP70 digested with XbaI and BamHI from pVR1012-mE7/MtHSP70

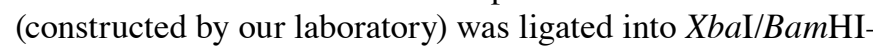
digested pVR1012-SigmE7. All constructs were validated by restriction enzyme digestion and DNA sequencing. Plasmid DNA was prepared with EndoFree Plasmid Purification kits from Qiagen Inc. (Valencia, CA, USA) resuspended in endotoxin-free normal saline at a concentration of $1 \mu \mathrm{g} / \mu \mathrm{l}$. The integrity of the DNA plasmids was verified by electrophoresis on a $1 \%$ agarose gel. DNA concentration was determined by absorbance measured at $260 \mathrm{~nm}$.

Western blot analysis. COS-7 cells with $70 \%$ confluence in a 6-well plate were transfected with $10 \mu \mathrm{g}$ plasmid DNA using Lipofectamine 2000 (Invitrogen). Supernatants and cells were harvested $48 \mathrm{~h}$ after transfection. The cells were lysed in $50 \mathrm{mM}$ Tris $\mathrm{HCl}, 150 \mathrm{mM} \mathrm{NaCl}, 1 \mathrm{mM}$ EDTA, $1 \%$ NP-40 and protease inhibitors. The protein concentration was determined using the BCA protein assay kit (Pierce). Each lysate $(60 \mu \mathrm{g})$ or $40 \mu \mathrm{l}$ of 4 -fold concentrated supernatants was denatured at $100^{\circ} \mathrm{C}$ for $5 \mathrm{~min}$, loaded on a $10 \%$ SDS-PAGE gel separated under reducing conditions, and transferred to polyvinylidene difluoride membranes (Bio-Rad Laboratories, Hercules, CA, USA). Membranes were blocked overnight with $4 \%$ BSA and incubated with polyclonal rabbit anti-HPV 16 E7 antibody $(1: 2,000)$ followed by horseradish peroxidase-conjugated goat anti-rabbit IgG (1:10,000; Beijing Zhongshan Golden Bridge Biotechnology Co., Ltd., Beijing, China). Blots were developed by chemiluminescence reagent (ECL kit; Pierce).

Mice and tumor cell line. Six- to 8-week-old female C57BL/6 mice were purchased from the Institute of Zoology, Chinese Academy Sciences, and were maintained at the animal facility of the Institute of Laboratory Animal Sciences, Chinese Academy of Medical Sciences and Peking Union Medical College. All experimental protocols were approved by the Institutional Animal Care and Use Committee. Care was taken to minimize pain and discomfort to all animals during the procedures in the present study. TC- 1 cells were generated by co-transfection of primary pulmonary epithelial cells from C57BL/6 mice with HPV16 E6 and E7 and activated c-Ha-ras oncogenes. The cells were grown in RPMI-1640 supplemented with $10 \%$ fetal calf serum, $2 \mathrm{mM}$ L-glutamine, $1 \mathrm{mM}$ sodium pyruvate, $2 \mathrm{mM}$ non-essential amino acids and $0.4 \mathrm{mg} / \mathrm{ml}$ G418 and antibiotics.

DNA vaccination. The mice were divided into three groups ( $n=7$ per group). The mice were injected intramuscularly (i.m.) with $125 \mu \mathrm{g}$ bupivacaine hydrochloride into each side of the $\mathrm{M}$. quadriceps. One day later, the mice were inoculated with $50 \mu \mathrm{g}$ of DNA at the same site on each side of the M. quadriceps. One week later, the mice received the DNA constructs similar to the priming. All DNA constructs for injection were prepared with EndoFree Plasmid Purification kits from Qiagen.

ELISPOT assay. The ELISPOT assay was performed as described in our previous study (23). Briefly, 96-well ELISPOT plates (BD Pharmingen, San Diego, CA, USA) were coated with $5 \mu \mathrm{g} / \mathrm{ml}$ rat anti-mouse IFN- $\gamma$ antibody in $100 \mu \mathrm{l}$ of PBS. After overnight incubation at $4^{\circ} \mathrm{C}$, the wells were washed and blocked with RPMI-1640 culture medium containing $10 \%$ fetal calf serum. Different concentrations of freshly isolated splenocytes from each vaccinated mouse group (from $1 \times 10^{6}$ to $1.25 \times 10^{5} /$ well) were added to the wells along with $50 \mathrm{IU} / \mathrm{ml}$ IL-2 and $1 \mu \mathrm{g} / \mathrm{ml}$ E7 peptide containing CTL epitope $\left(\mathrm{H}-2 \mathrm{D}^{\mathrm{b}}\right.$, aa 49-57) (26). After a 24-h culture, the plate was washed followed by incubation with $2.5 \mu \mathrm{g} / \mathrm{ml}$ biotinylated IFN- $\gamma$ antibodies in $100 \mu 1$ in PBS containing $10 \%$ fetal calf serum at $4^{\circ} \mathrm{C}$ overnight. After washing, avidin-HRP in $100 \mu \mathrm{l}$ of PBS was added and incubated for $1 \mathrm{~h}$ at room temperature. After washing five times, spots were developed by adding $100 \mu \mathrm{l}$ AEC (3-amino-9-ethylcarbazole) solution. The spots were counted using an ELISPOT Reader system.

Intracytoplasmic cytokine staining and flow cytometric analysis. To detect E7-specific $\mathrm{CD}^{+} \mathrm{T}$ cell precursors and E7-specific $\mathrm{CD}^{+}{ }^{+} \mathrm{T}$ helper cell responses, splenocytes from each vaccinated mouse group were incubated either with $2 \mu \mathrm{g} / \mathrm{ml}$ of E7 peptide (aa 49-57) or with $2 \mu \mathrm{g} / \mathrm{ml}$ of E7 peptide (aa 30-67) 
containing MHC class II epitope (27) for $20 \mathrm{~h}$. Golgistop (BD Pharmingen) was added $6 \mathrm{~h}$ before harvesting the cells from the culture. Cells were then washed once in FACScan buffer and stained with phycoerythrin (PE)-conjugated monoclonal rat anti-mouse CD8 or CD4 antibody (BD Pharmingen). Cells were subjected to intracellular cytokine staining using the Cytofix/Cytoperm kit according to the manufacturer's instructions (BD Pharmingen). PE-conjugated anti-IFN- $\gamma$ or anti-IL-4 antibodies and the FITC-conjugated rat IgG2a, $\mathrm{k}$ or PE-conjugated at IgG1 isotype control antibody were all purchased from Pharmingen. Analyses were performed on a Beckman Coulter EPICS XL (Beckman Coulter Inc., Fullerton, CA, USA).

Anti-E7 ELISA. The anti-HPV16E7 antibodies in the sera were determined by a direct ELISA as previously described $(23,25)$. Serially diluted sera collected from mice on day 10 postimmunization were incubated at $4^{\circ} \mathrm{C}$ overnight with $100 \mathrm{ng}$ of bacteria-derived HPV16 E7 protein in an ELISA plate. A 1:3,000 dilution of HRP-conjugated goat anti-mouse IgG antibody (Beijing Zhongshan Golden Bridge Biotechnology) was used. The ELISA plate was read using a standard ELISA reader at $490 \mathrm{~nm}$.

In vivo tumor protection experiments. For the tumor protection experiment, C57BL/6 mice (7 per group) were vaccinated i.m. with $100 \mu \mathrm{g}$ of pVR1012-mE7/MtHSP70, pVR1012-SigmE7/ MtHSP70 or pVR1012 vector control twice with a 1-week interval. One week after the last vaccination, mice were challenged s.c. with $7.5 \times 10^{4} \mathrm{TC}-1$ cells per mouse in the right flank and then monitored twice a week for tumor growth.

In vivo tumor treatment experiments. To test the ability of the DNA vaccination to inhibit the growth of established tumors, C57BL/6 mice (7 per group) were s.c. challenged with $7.5 \times 10^{4}$ TC-1 cells per mouse in the right flank. Three days later, mice were immunized with $100 \mu \mathrm{g}$ of each plasmid of pVR1012mE7/MtHSP70, pVR1012-SigmE7/MtHSP70 and pVR1012 i.m., and the mice were boosted 1 week after the first immunization. Mice were monitored twice a week for tumor growth.

Data analyses. ELISPOT and FACS data were analyzed using the mean of two sample comparison of Poisson distribution. ELISA data were analyzed using the Student's t-test, and tumor incidence data were analyzed by the Fisher's exact probabilities in a $2 \times 2$ table. Values of $\mathrm{P}<0.05$ were considered to indicate a statistically significant result.

\section{Results}

Detection of fusion proteins secreted from transfected cells in vitro. As determined by the western blot results (Fig. 1A and B) mE7 and HSP70 fusion proteins were confirmed to be expressed both in the culture supernatants and in the cell lysates of cells after transfection with mE7/MtHSP70 or SigmE7/MtHSP70 fusion DNA constructs, whereas cells transfected with pVR1012 showed no expression signal in the supernatants and in the lysates. $\beta$-actin was detected as the internal loading control (Fig. 1A). The ratio of mE7/MtHSP70 or SigmE7/MtHSP70 fusion protein vs. $\beta$-actin was 1.21 and
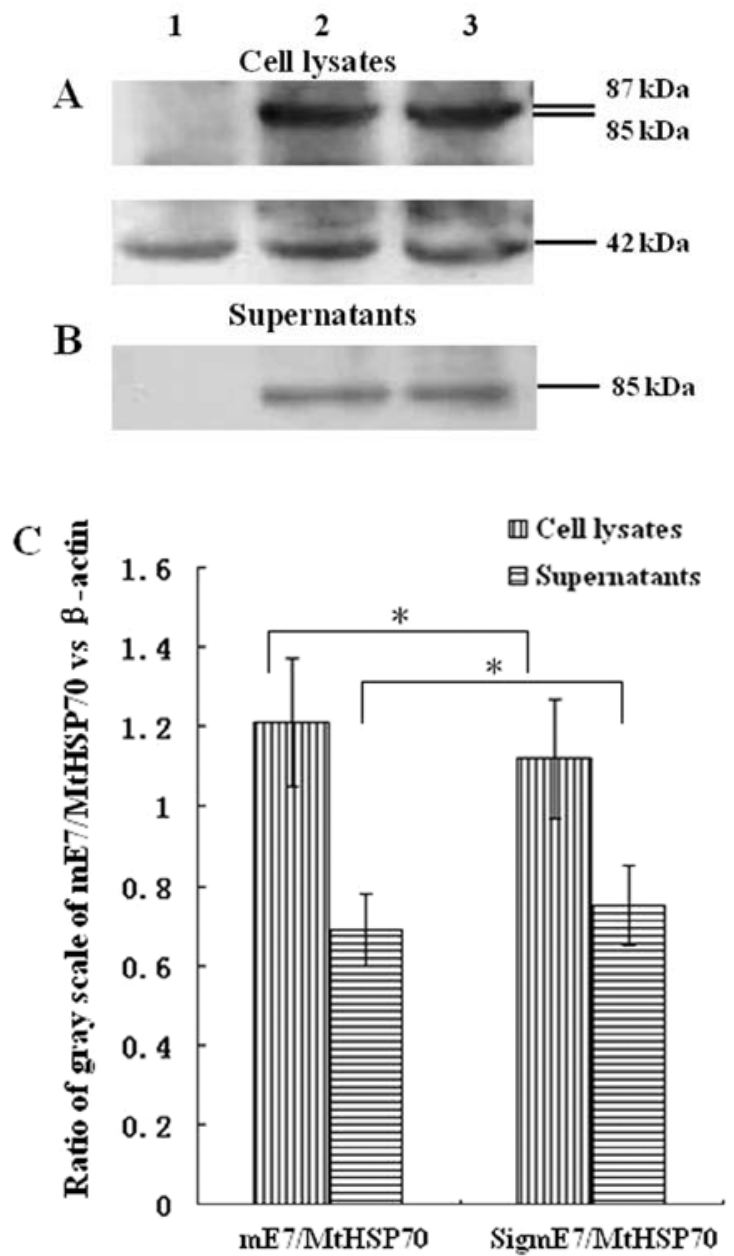

Figure 1. Expression level in COS-7 cells following transfection with different DNA constructs. (A and B) Western blotting of the fusion protein expression. Lane 1, pVR1012; lane 2, pVR1012-mE7/MtHSP70; and lane 3, pVR1012-SigmE7/MtHSP70. Cell lysates and culture supernatants were blotted with a polyclonal rabbit anti-HPV16 E7 antibody. The relative molecular weights of mE7/MtHSP70, SigmE7/MtHSP70 and $\beta$-actin are $\sim 85,87$ and $42 \mathrm{kDa}$, respectively. (C) Gray scale scanning analysis of the ratios of mE7/MtHSP70 and SigmE7/MtHSP70 to $\beta$-actin. The ratios of the gray scale of mE7/MtHSP70 or mE7/MtHSP70 to $\beta$-actin were similar both in the cell lysates and in the culture supernatants ( $(\mathrm{P}>0.05)$. Results shown here are from experiments performed three times.

1.12 in the cell lysates as determined by gray scale scanning analysis from a gel imaging system (Fig. 1C). No difference was observed in the mE7/MtHSP70 fusion protein expression level between the cell lysates of cells transfected with the SigmE7/MtHSP70 and mE7/MtHSP70 fusion DNA constructs (P>0.05). The ratios of $\mathrm{mE7/MtHSP70}$ or SigmE7/MtHSP70 fusion protein to $\beta$-actin were 0.69 and 0.75 in the culture supernatants (Fig. 1C); no significant difference was observed between the values $(P>0.05)$. These results indicate that addition of a signal peptide at the upstream of the mE7/MtHSP70 fusion gene did enhance the secretary expression of the $\mathrm{mE7}$ and MtHSP70 fusion protein.

mE7/MtHSP70 fusion DNA induces a higher level of E7-specific CD8 $8^{+}$T cells than SigmE7/MtHSP70 DNA. CD8 $\mathrm{T}$ lymphocytes are one of the most critical components among antitumor effectors in tumor immunity. Thus, we examined E7-specific $\mathrm{CD} 8^{+} \mathrm{T}$-cell precursor frequency induced by DNA 
A
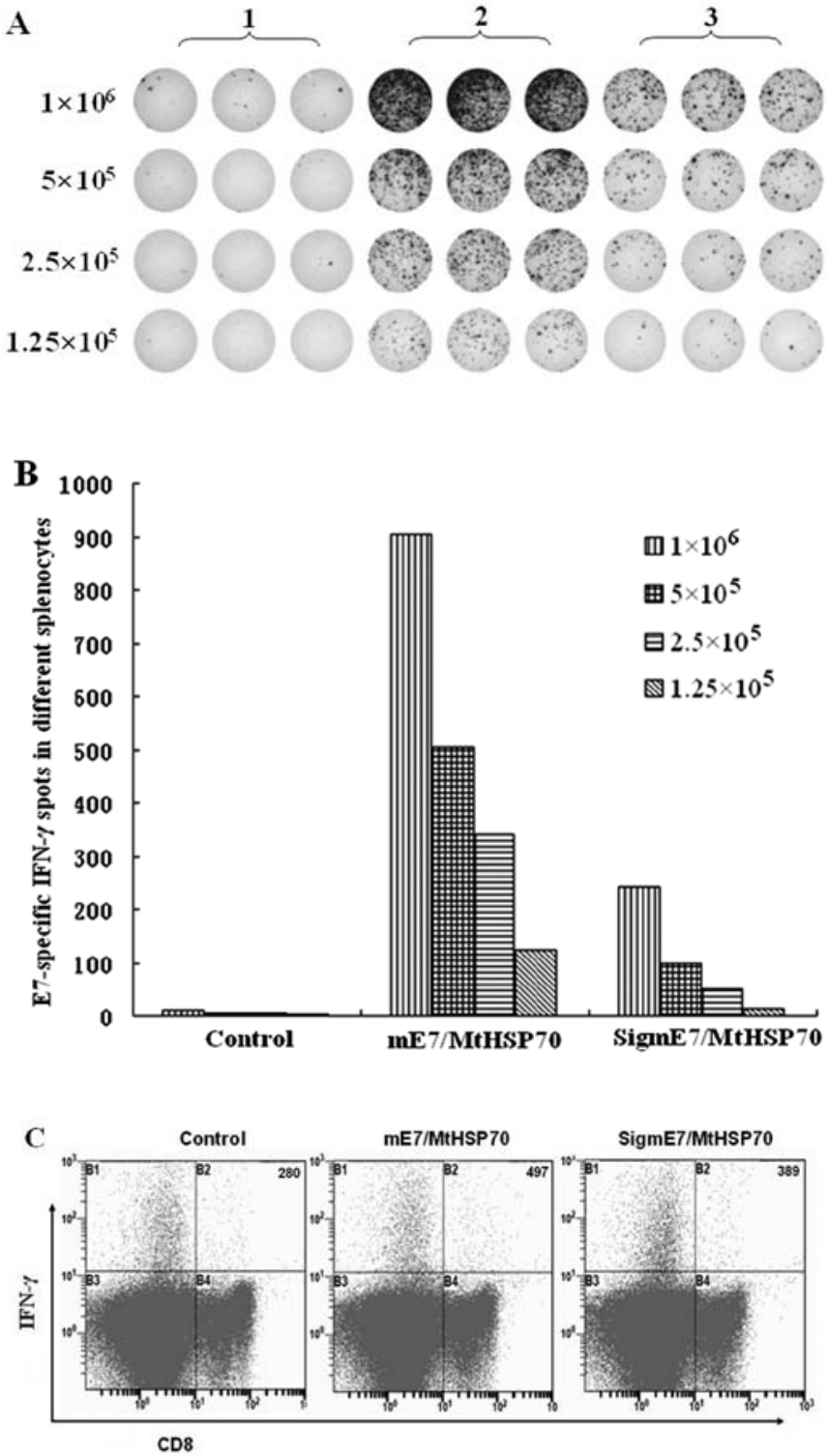

Figure 2. E7-specific $\mathrm{CD}^{+} \mathrm{T}$-cell precursors in C57BL/6 mice vaccinated with the recombinant DNA vaccines: pVR1012 (Control), pVR1012mE7/MtHSP70 (mE7/MtHSP70) and pVR1012-SigmE7/MtHSP70 (SigmE7/MtHSP70). (A and B) ELISPOT assay (as described in Materials and methods). (A) A spot image of different numbers of splenocytes from each vaccinated mouse group as photographed by the ELISPOT reader system. Different numbers of splenocytes from the indicated mouse group: 1, Control group; 2, mE7/MtHSP70 group; 3, SigmE7/MtHSP70 group. (B) Graph of E7-specific IFN- $\gamma$ spots counted. The spot numbers are the means of triplicates \pm SE in each vaccinated group at a different cell numbers. (C) Flow cytometric analysis of IFN- $\gamma$ secreting E7-specific $\mathrm{CD}^{+}$cells in mice vaccinated with various DNA vaccines (see Materials and methods). The number of $\mathrm{CD}^{+}$and IFN- $\gamma^{+}$double-positive cells in $3 \times 10^{5}$ splenocytes are indicated in the upper right corner. ELISPOT data and intracellular cytokine staining shown here are from one representative experiment of two performed tests.

vaccination. ELISPOT results are shown in Fig. 2A and B. The number of E7-specific IFN- $\gamma$-producing $\mathrm{CD}^{+} \mathrm{T}$ cells in splenocytes from the mE7/MtHSP70 fusion DNA immunized mice was greater than 4 times that from the SigmE7/ MtHSP70 fusion DNA immunized mice (503 vs. 100 per $5 \times 10^{5}$ splenocytes; $\mathrm{P}<0.01)$. Results of the flow cytometric analysis of E7-specific IFN- $\gamma$-producing $\mathrm{CD}^{+}{ }^{+} \mathrm{T}$ cells are shown in Fig. $2 \mathrm{C}$.

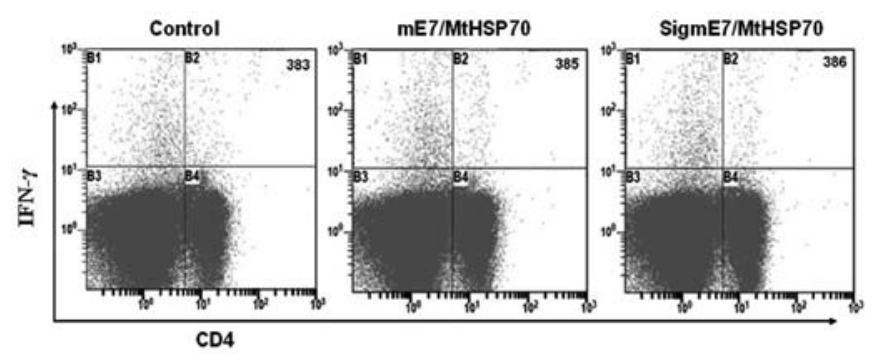

Figure 3. Flow cytometric analysis of IFN- $\gamma$-secreting E7-specific $\mathrm{CD} 4^{+} \mathrm{T}$ cells in mice vaccinated with the recombinant DNA vaccines. C57BL/6 mice were immunized as described in Fig. 2. Splenocytes from the vaccinated mice were cultured in vitro with the E7 peptide (aa 30-67) overnight and were stained for both CD4 and intracellular IFN- $\gamma$. The number of IFN- $\gamma$ secreting $\mathrm{CD}^{+} \mathrm{T}$ cells was analyzed by flow cytometry. The numbers of $\mathrm{CD} 4^{+} / \mathrm{IFN}-\gamma$ double positive $\mathrm{T}$ cells in $3 \times 10^{5}$ splenocytes are indicated in the upper right corner. The data of the intracellular cytokine staining shown here are one representative experiment of two experiments performed.

Subtracting the background produced by the control ( 280 cells $/ 3 \times 10^{5}$ splenocytes), mice vaccinated with $\mathrm{mE} 7 / \mathrm{MtHSP} 70$ fusion DNA generated the highest number of IFN- $\gamma$-secreting $\mathrm{CD}^{+} \mathrm{T}$-cell precursors (217 cells $/ 3 \times 10^{5}$ splenocytes), whereas mice vaccinated with SigmE7/MtHSP70 fusion DNA generated only $\sim 109$ IFN- $\gamma$-producing $\mathrm{CD} 8^{+} \mathrm{T}$-cell precursors per $3 \times 10^{5}$ splenocytes $(\mathrm{P}<0.01)$. The results were correlated closely with that of the ELISPOT results (Fig. 2A and B). The results indicated that while MtHSP70 or Sig/MtHSP70 linked to mE7 could both induce the activation of antigen-specific $\mathrm{CD} 8^{+} \mathrm{T}$ cells, mE7/MtHSP70 induced a higher level of E7-specific $\mathrm{CD}^{+} \mathrm{T}$ cell response than SigmE7/MtHSP70 DNA.

Neither the mE7/MtHSP70 nor SigmE7/MtHSP70 fusion DNA vaccine elicits E7-specific $C D 4^{+} \mathrm{T}$ cell-mediated immune responses. To determine the $\mathrm{E} 7$-specific $\mathrm{CD}^{+}{ }^{+} \mathrm{T}$-precursors activated by the vaccines, we performed double staining for the CD4 surface marker and the intracellular IFN- $\gamma$ or IL-4 in the splenocytes after incubation with the E7 peptide (aa 30-67). Results are shown in Fig. 3. There was no significant difference in the number of E7-specific IFN- $\gamma$-secreting CD4 cells as determined using flow cytometry staining among the various vaccination groups, and no significant $\mathrm{CD} 4^{+} / \mathrm{IL}-4^{+}$ double-positive cells were identified in mice receiving $\mathrm{mE7/}$ MtHSP70, SigmE7/MtHSP70 and control DNA (data not shown). These data indicated that neither mE7/MtHSP70 nor SigmE7/MtHSP70 fusion DNA vaccine activated E7-specific Th cell responses.

Neither mE7/MtHSP70 nor SigmE7/MtHSP70 fusion DNA vaccine induces E7-specific antibodies. No E7-specific antibodies were detected in the sera of mice in any of the vaccinated groups (Fig. 4). The results suggest that the modifications introduced in the construction of the two DNA vaccines could not elicit antibody responses.

mE7/MtHSP70 and SigmE7/MtHSP70 fusion DNA vaccines prevent tumors in vivo. As shown Fig. 5, both mE7/MtHSP70 and SigmE7/MtHSP70 fusion DNA vaccines induced effective immunity against TC-1 tumors. At $\sim 2$ months after TC-1 tumor challenge, $100 \%$ of the mice receiving $\mathrm{mE7} / \mathrm{MtHSP} 70$ 


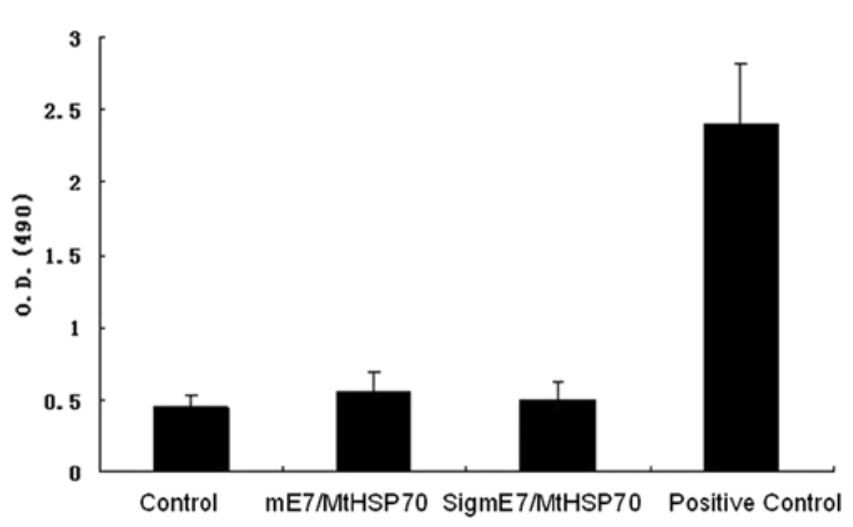

Figure 4. E7-specific antibody responses in C57BL/6 mice immunized with the recombinant DNA vaccines. C57BL/6 mice were immunized i.m. with pVR1012 (Control), pVR1012-mE7/MtHSP70 (mE7/MtHSP70) and pVR1012-SigmE7/MtHSP70 (SigmE7/MtHSP70). Serum samples were obtained from immunized mice 10 days after vaccination. Rabbit anti-E7 antibody was used as a positive control. The presence of the E7-specific antibody was detected by ELISA using a serial dilution of sera. The results from the 1:20 dilution are presented showing the mean absorbance (A490 nm) \pm SE.

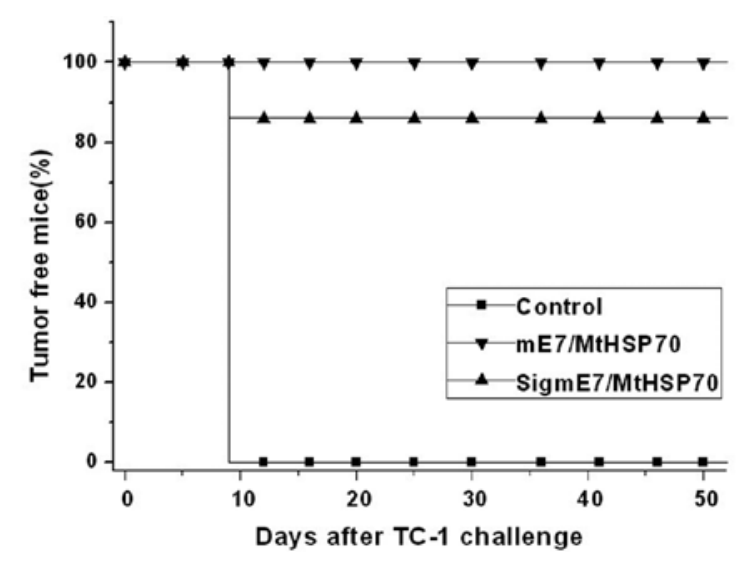

Figure 5. In vivo tumor prevention experiments. The mE7/MtHSP70 DNA vaccine protects mice against TC-1 tumor growth more effectively than the SigmE7/MtHSP70 DNA vaccine. C57BL/6 mice (7 mice per group) were immunized i.m. twice with $100 \mu \mathrm{g}$ of each DNA construct: pVR1012-mE7/MtHSP70 (mE7/MtHSP70), pVR1012-SigmE7/MtHSP70 (SigmE7/MtHSP70) and the pVR1012 (Control), twice with a 1-week interval. One week after the second vaccination, the mice were challenged s.c. with $7.5 \times 10^{4} \mathrm{TC}-1$ cells in the right flank, and were monitored for tumor growth twice a week. The results shown here are from a representative experiment of two experiments performed.

fusion DNA remained tumor free, whereas only $86 \%$ of mice receiving SigmE7/MtHSP70 fusion DNA remained tumor free. In contrast, all of the mice receiving the empty vector developed a tumor growth on day 12 after tumor challenge. The results demonstrated that the mE7/MtHSP70 fusion DNA vaccine induced a much stronger antitumor immune effect, indicating that addition of a secretary signal peptide at the $\mathrm{N}$ terminal of the $\mathrm{mE7} / \mathrm{MtHSP70}$ fusion protein did not enhance the immune effect of the mE7/MtHSP70 antigen.

mE7/MtHSP70 and SigmE7/MtHSP70 fusion DNA vaccines eradicate tumors in vivo. The therapeutic effects of the two DNA vaccines in eradicating the established TC-1 tumors are

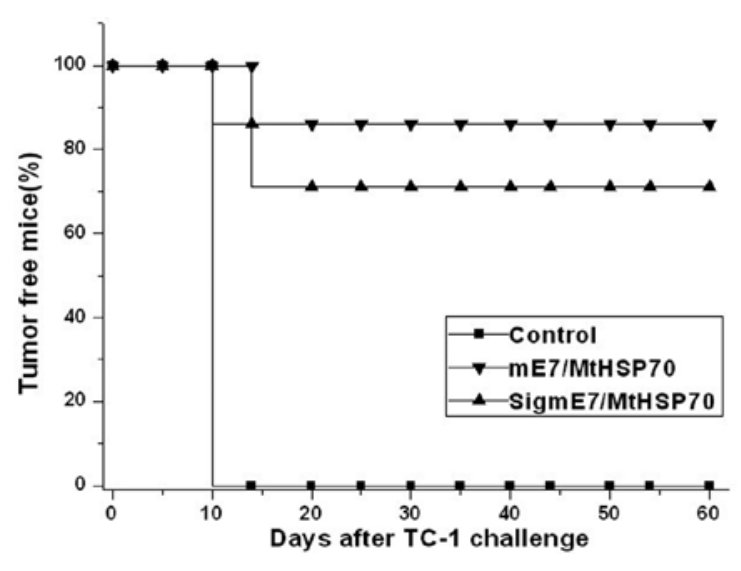

Figure 6. In vivo tumor treatment experiments. The mE7/MtHSP70 DNA vaccine elicits stronger antitumor immunity than the SigmE7/MtHSP70 DNA vaccine. Each mouse was initially challenged s.c. with $7.5 \times 10^{4} \mathrm{TC}-1$ cells in the right flank and then was vaccinated i.m. twice with $100 \mu \mathrm{g}$ of each DNA construct: pVR1012-mE7/MtHSP70 (mE7/MtHSP70), pVR1012SigmE7/MtHSP70 (SigmE7/MtHSP70) and pVR1012 (Control), twice with a 1-week interval. The mice were monitored for tumor growth twice a week. The results shown here are from a representative experiment of two expreriments performed.

shown in Fig. 6. After immunization, 100\% mice receiving the empty vector developed tumors soon after TC-1 cell challenge. In contrast, $71 \%$ mice receiving the SigmE7/MtHSP70 fusion DNA vaccine were tumor free, and the percentage of tumorfree mice receiving the $\mathrm{mE7/MtHSP70}$ fusion DNA vaccine was up to $86 \%$ for at least 2 months. The results showed that the vaccination with either forms of mE7 and MtHSP70 fusion DNA vaccines could eradicate the established tumors, and the $\mathrm{mE7} / \mathrm{MtHSP} 70$ fusion DNA vaccine induced stronger antitumor activity than the SigmE7/MtHSP70 fusion DNA vaccine. The above results indicate that addition of a secretary signal peptide at the $\mathrm{N}$ terminal of the mE7/MtHSP70 fusion protein did not enhance the immune effect of the $\mathrm{mE7/MtHSP70} \mathrm{fusion} \mathrm{DNA} \mathrm{vaccine.}$

\section{Discussion}

Our results showed that antitumor activity of the two mE7 and MtHSP70 fusion DNA vaccines was mainly dependent on E7-specific $\mathrm{CD}^{+} \mathrm{T}$ cell responses, whereas $\mathrm{CD} 4^{+} \mathrm{T}$ cells and the E7-specific antibody were not detected, indicating that the antigen presentation pathway involved in the $\mathrm{CD} 8^{+} \mathrm{T}$ cell responses are mainly mediated by MHC class I molecule participating in the antigen presentation pathway. Our results were consistent with previous reports that specific $\mathrm{CD} 8^{+} \mathrm{T}$ cell responses generated by antigen and the HSP70 fusion gene were independent of the help of $\mathrm{CD}^{+} \mathrm{T}$ cells $(11,28)$. HSP70 plays an important role, not only in the process of protein folding, transport and degradation, but also in the participation of directing more efficient antigen presentation to $\mathrm{CD}^{+} \mathrm{T}$ cells through the MHC class I pathway (20,29-31). The possible mechanisms of HSP70 enhancement of $\mathrm{CD}^{+} \mathrm{T}$ cell responses independent of the help of $\mathrm{CD}^{+} \mathrm{T}$ cells may involve activation of DCs directly and indirectly to release proinflammatory cytokines, cross presentation and the intrinsic molecular chaperone activity of HSP70. 
HSP70 was first considered as a cytoplasmic protein. Afterwards, it was found that HSP70 can also be released from cells and become an extracellular protein, playing versatile biological functions. In the present study, expression of the mE7/MtHSP70 fusion protein was observed both in the cell lysates and in culture supernatants after transfection with either mE7/MtHSP70 or SigmE7/MtHSP70 fusion DNA constructs. To our surprise, $\mathrm{mE7/MtHSP70} \mathrm{fusion} \mathrm{proteins} \mathrm{can} \mathrm{be} \mathrm{secreted}$ from cells efficiently even without the guidance of a signal peptide. Why could HSP70 be released from cells? At first, release of HSP70 was considered to be a pathologic phenomenon, i.e. it can be released from cells during cellular necrosis and cytolysis (32). Further studies found that several human cancer cell lines could also secrete HSP70, and the secretion can be increased when these cells are transfected with HSP70 DNA (33). Some specific cell membrane microdomains, for example, exosome and endosome lysosomes, may play important roles in HSP70 exocytosis (34-36). Our results showed that HSP70 and the antigen fusion protein can be secreted from cells, and addition of a heterogeneous signal peptide at the $\mathrm{N}$ terminal of mE7/MtHSP70 did not enhance secretion of the fusion protein, indicating that HSP70 has potent intrinsic secretion activity and can direct HSP70 and antigen fusion protein out of the cells via several types of secretary pathways.

The reason why the mE7/MtHSP70 fusion DNA vaccine produced a more effective $\mathrm{CD} 8^{+} \mathrm{T}$ cell response and antitumor activity than the SigmE7/MtHSP70 vaccine can be explained by the following three reasons. Firstly, in the antigen direct presentation pathway, DCs intake DNA construct and express $\mathrm{mE7/MtHSP70} \mathrm{fusion} \mathrm{protein,} \mathrm{providing} \mathrm{a} \mathrm{favorable} \mathrm{advan-}$ tage for HSP70 and the antigen to function in the same DC. Thus, HSP70 may have an opportunity to chaperone E7 peptide cross-presentation to $\mathrm{CD}^{+} \mathrm{T}$ cells by the MHC class I pathway. HSPs have also been proposed to be involved in processing MHC class I restricted antigens $(29,37,38)$. How HSP70 takes chaperone effects in the form of the mE7/HSP70 fusion protein, deserves further study. In this case, the existence of a signal peptide in the fusion protein may influence the chaperone activity of HSP70. This may explain the reason why the SigmE7/MtHSP70 fusion DNA vaccine had decreased potency compared with the mE7/MtHSP70 fusion DNA vaccine. Secondly, in the antigen cross-presentation pathway, when muscle cells and/or DCs intake the DNA construct, then the synthesize and release the coded protein out of cells. The secretory protein is then taken up by DCs via mediation of the endocytic receptors, and present the antigen peptide to $\mathrm{CD} 8^{+}$ $\mathrm{T}$ cells. When then $\mathrm{mE7/MtHSP70}$ fusion protein is secreted from the cells transfected with the SigmE7/MtHSP70 fusion DNA vaccine, the signal peptide will be cut off from the fusion protein, so the secretory fusion proteins produced by the $\mathrm{mE7/}$ MtHSP70 or SigmE7/MtHSP70 fusion DNA vaccines are the same, both in the form of mE7/MtHSP70. As the secretory levels of mE7/HSP70 fusion proteins from cells transfected with SigmE7/MtHSP70 and mE7/MtHSP70 fusion DNA vaccines were similar, we could not explain the different $\mathrm{CD}^{+} \mathrm{T}$ cell responses of these two DNA vaccines from this aspect. Lastly, another possible condition, is that when the muscle cells which take up the two DNA vaccines are dead, the fusion proteins are released out of the cells in the form of either SigmE7/MtHSP70 or mE7/MtHSP70. In this case, the existence of the signal peptide may interfere with the binding of HSP70 to its endocytic receptor on DCs resulting in the decreased immunogenicity of the SigmE7/MtHSP70 fusion DNA vaccine.

In summary, we constructed a more effective and simple HPV 16 therapeutic DNA vaccine that is capable of generating significantly high levels of antigen-specific antitumor activity without the addition of a signal peptide gene sequence. Our observations may serve as an important foundation and significant reference for future basic research and clinical trials.

\section{Acknowledgements}

The present study was supported by a grant from the National Natural Science Foundation of China (no. 30271355), the National Natural Science Foundation of China (no. 31070 813) and the Nature Science Foundation for Young Scholars of Shandong Province (ZR2010HQ009).

\section{References}

1. Jemal A, Bray F, Center MM, Ferlay J, et al: Global cancer statistics. CA Cancer J Clin 61: 69-90, 2011.

2. Clifford GM, Smith JS, Plummer M, et al: Human papillomavirus types in invasive cervical cancer worldwide: a meta-analysis. $\mathrm{Br}$ J Cancer 88: 63-73, 2003.

3. Quek SC, Lim BK, Domingo E, et al: Human papillomavirus type distribution in invasive cervical cancer and high-grade cervical intraepithelial neoplasia across 5 countries in Asia. Int J Gynecol Cancer 23: 148-156, 2013.

4. Brown DR, Kjaer SK, Sigurdsson K, et al: The impact of quadrivalent human papillomavirus (HPV; types $6,11,16$, and 18) L1 virus-like particle vaccine on infection and disease due to oncogenic nonvaccine HPV types in generally HPV-naive women aged 16-26 years. J Infect Dis 199: 926-935, 2009.

5. Kemp TJ, Hildesheim A, Safaeian M, et al: HPV16/18 L1 VLP vaccine induces cross-neutralizing antibodies that may mediate cross-protection. Vaccine 29: 2011-2014, 2011.

6. Chen $\mathrm{CH}$, Wang TL, Hung CF, et al: Boosting with recombinant vaccinia increases HPV-16 E7-specific T cell precursor frequencies of HPV-16 E7-expressing DNA vaccines. Vaccine 18: 2015-2022, 2000.

7. Brinkman JA, Xu X and Kast WM: The efficacy of a DNA vaccine containing inserted and replicated regions of the E7 gene for treatment of HPV-16 induced tumors. Vaccine 25: 3437-3444, 2007.

8. Eiben GL, Velders MP, Schreiber H, et al: Establishment of an HLA-A*0201 human papillomavirus type 16 tumor model to determine the efficacy of vaccination strategies in HLA-A*0201 transgenic mice. Cancer Res 62: 5792-5799, 2002.

9. Ohlschlager P, Pes M, Osen W, et al: An improved rearranged human papillomavirus type 16 E7 DNA vaccine candidate (HPV-16 E7SH) induces an E7 wildtype-specific T cell response. Vaccine 24: 2880-2893, 2006.

10. Gurunathan S, Klinman DM and Seder RA: DNA vaccines: immunology, application, and optimization. Annu Rev Immunol 18: 927-974, 2000

11. Chen CH, Wang TL, Hung CF, et al: Enhancement of DNA vaccine potency by linkage of antigen gene to an HSP70 gene. Cancer Res 60: 1035-1042, 2000.

12. Li Y, Subjeck J, Yang G, et al: Generation of anti-tumor immunity using mammalian heat shock protein 70 DNA vaccines for cancer immunotherapy. Vaccine 24: 5360-5370, 2006.

13. Hauser H, Shen L, Gu QL, et al: Secretory heat-shock protein as a dendritic cell-targeting molecule: a new strategy to enhance the potency of genetic vaccines. Gene Ther 11: 924-932, 2004.

14. Milani V, Noessner E, Ghose S, et al: Heat shock protein 70 : role in antigen presentation and immune stimulation. Int $\mathrm{J}$ Hyperthermia 18: 563-575, 2002.

15. Li Z, Menoret A and Srivastava P: Roles of heat-shock proteins in antigen presentation and cross-presentation. Curr Opin Immunol 14: 45-51, 2002.

16. Bendz H, Ruhland SC, Pandya MJ, et al: Human heat shock protein 70 enhances tumor antigen presentation through complex formation and intracellular antigen delivery without innate immune signaling. J Biol Chem 282: 31688-31702, 2007. 
17. Udono $\mathrm{H}$, Ichiyanagi $\mathrm{T}$, Mizukami S, et al: Heat shock proteins in antigen trafficking - implications on antigen presentation to $\mathrm{T}$ cells. Int J Hyperthermia 25: 617-625, 2009.

18. Srivastava P: Interaction of heat shock proteins with peptides and antigen presenting cells: chaperoning of the innate and adaptive immune responses. Annu Rev Immunol 20: 395-425, 2002.

19. Osterloh A and Breloer M: Heat shock proteins: linking danger and pathogen recognition. Med Microbiol Immunol 197: 1-8, 2008.

20. Binder RJ and Srivastava PK: Peptides chaperoned by heat-shock proteins are a necessary and sufficient source of antigen in the cross-priming of $\mathrm{CD}^{+} \mathrm{T}$ cells. Nat Immunol 6: 593-599, 2005.

21. Massa C, Guiducci C, Arioli I, et al: Enhanced efficacy of tumor cell vaccines transfected with secretable hsp70. Cancer Res 64: 1502-1508, 2004.

22. Murshid A, Gong J, Stevenson MA, et al: Heat shock proteins and cancer vaccines: developments in the past decade and chaperoning in the decade to come. Expert Rev Vaccines 10: 1553-1568, 2011.

23. Zong J, Peng Q, Wang Q, et al: Human HSP70 and modified HPV16 E7 fusion DNA vaccine induces enhanced specific CD8 $\mathrm{T}$ cell responses and anti-tumor effects. Oncol Rep 22: 953-961, 2009.

24. Trimble CL, Peng S, Kos F, et al: A phase I trial of a human papillomavirus DNA vaccine for HPV $16^{+}$cervical intraepithelial neoplasia 2/3. Clin Cancer Res 15: 361-367, 2009.

25. Wang QY, Xu YF, Fan DS, et al: Linkage of modified human papillomavirus type $16 E 7$ to CD40 ligand enhances specific CD8 ${ }^{+}$T-lymphocyte induction and anti-tumour activity of DNA vaccine. Zhongguo Yi Xue Ke Xue Yuan Xue Bao 29: 584-591, 2007 (In Chinese).

26. Feltkamp MC, Smits HL, Vierboom MP, et al: Vaccination with cytotoxic $\mathrm{T}$ lymphocyte epitope-containing peptide protects against a tumor induced by human papillomavirus type 16-transformed cells. Eur J Immunol 23: 2242-2249, 1993.

27. Tindle RW, Fernando GJ, Sterling JC, et al: A 'public' T-helper epitope of the E7 transforming protein of human papillomavirus 16 provides cognate help for several E7 B-cell epitopes from cervical cancer-associated human papillomavirus genotypes. Proc Natl Acad Sci USA 88: 5887-5891, 1991.
28. Hsu KF, Hung CF, Cheng WF, et al: Enhancement of suicidal DNA vaccine potency by linking Mycobacterium tuberculosis heat shock protein 70 to an antigen. Gene Ther 8: 376-383, 2001.

29. Liu H, Wu BH, Rowse GJ, et al: Induction of CD4-independent E7-specific $\mathrm{CD}^{+}$memory response by heat shock fusion protein. Clin Vaccine Immunol 14: 1013-1023, 2007.

30. Huang Q, Richmond JF, Suzue K, et al: In vivo cytotoxic T lymphocyte elicitation by mycobacterial heat shock protein 70 fusion proteins maps to a discrete domain and is $\mathrm{CD} 4^{+} \mathrm{T}$ cell independent. J Exp Med 191: 403-408, 2000.

31. Delneste Y, Magistrelli G, Gauchat J, et al: Involvement of LOX-1 in dendritic cell-mediated antigen cross-presentation. Immunity 17: 353-362, 2002.

32. Saito K, Dai Y and Ohtsuka K: Enhanced expression of heat shock proteins in gradually dying cells and their release from necrotically dead cells. Exp Cell Res 310: 229-236, 2005.

33. Wang MH, Grossmann ME and Young CY: Forced expression of heat-shock protein 70 increases the secretion of Hsp70 and provides protection against tumour growth. $\mathrm{Br} \mathrm{J}$ Cancer 90: 926-931, 2004

34. Mambula SS, Stevenson MA, Ogawa K, et al: Mechanisms for Hsp70 secretion: crossing membranes without a leader. Methods 43: 168-175, 2007.

35. Broquet AH, Thomas G, Masliah J, et al: Expression of the molecular chaperone Hsp70 in detergent-resistant microdomains correlates with its membrane delivery and release. J Biol Chem 278: 21601-21606, 2003

36. Lancaster GI and Febbraio MA: Exosome-dependent trafficking of HSP70: a novel secretory pathway for cellular stress proteins. J Biol Chem 280: 23349-23355, 2005.

37. Srivastava PK, Udono H, Blachere NE, et al: Heat shock proteins transfer peptides during antigen processing and CTL priming. Immunogenetics 39: 93-98, 1994

38. Srivastava PK and Udono H: Heat shock protein-peptide complexes in cancer immunotherapy. Curr Opin Immunol 6: 728-732, 1994. 